\title{
Experimental Testing of Cooperative Adaptive Cruise Control using Small Electric Vehicles
}

\author{
Takeki Ogitsu ${ }^{\mathrm{a},{ }^{*}}$, Manabu Omae ${ }^{\mathrm{b}}$ \\ ${ }^{a}$ Gunma University, 1-5-1, Tenjin-cho, Kiryu-shi Gunma 376-8515, Japan

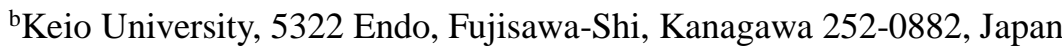 \\ *Corresponding Author: ogitsu@ gunma-u.ac.jp
}

\begin{abstract}
This paper reports on the development of a cooperative adaptive cruise control (CACC) algorithm for small electric vehicles (EVs), and on the evaluation of this algorithm through application to small EVs. In order to enhance the feasibility of autonomous vehicle driving systems, both ride comfort, which depends on longitudinal control, and stable vehicle-to-vehicle $(\mathrm{V} 2 \mathrm{~V})$ distance are essential. The CACC algorithm presented in this study has been developed to satisfy these technological requirements. Generally, as regards determining the state quantities of a preceding vehicle, methods that exploit information received from the preceding-vehicle internal sensors via $\mathrm{V} 2 \mathrm{~V}$ communication are more accurate than external-sensor-based technologies. The former approach is used by CACC, and such algorithms can achieve both ride comfort and stable V2V distance. However, to date, insufficient evaluation of the performance of such technologies has been conducted using actual vehicles. Therefore, the developed CACC for small EVs presented in this study is evaluated using several vehicles. In this paper, previous research on driving assistant systems such as CACC is first described. Then, studies related to the CACC presented in this work are introduced and their technological problems are identified. Next, the designed CACC algorithm is explained. Finally, the experimental procedure and results are presented and discussed, where the performance of four small EVs using the CACC algorithm developed in this study are examined. This evaluation confirms the capability of CACC to ensure both ride comfort and a stable $\mathrm{V} 2 \mathrm{~V}$ distance.
\end{abstract}

Keywords: Car-following system, cooperative adaptive cruise control, string stability, V2V communication, intelligent transport systems.

\section{Introduction}

Advanced driving assistance systems (ADAS) are receiving increased research attention and are being developed with increasing celerity. In general, ADAS refers to systems that perform driver tasks concerning recognition, judgement, and operation. Recently developed ADAS can not only provide information for drivers, but can also act as driver substitutes, using advanced technology based on sensors, actuators, and computers. Adaptive cruise control (ACC) and the Lane Keeping Assist System (LKAS) are representative examples of ADAS that can act as driver substitutes. ACC is a longitudinal controller that controls the target vehicle velocity, which is set by the driver, in the absence of preceding vehicles. In the presence of a preceding vehicle, the ACC follows that vehicle by targeting the driver-set vehicle-to-vehicle (V2V) time. In contrast, LKAS is a lateral controller that observes road lanes and controls the vehicle steering wheel to conform to those boundaries. A fully automated driving system that can act as a substitute to perform all driver tasks will be accomplished in the future, through the advancement and integration of the above systems.

However, some technological problems must be overcome prior to realization of a fully automated driving system. As regards longitudinal control, the simultaneous achievement of both ride comfort and $\mathrm{V} 2 \mathrm{~V}$ distance stability remains problematic for ACC. One technology developed to solve this problem is cooperative adaptive cruise control (CACC), an ACC that functions based on the preceding-vehicle state quantities, which are obtained via $\mathrm{V} 2 \mathrm{~V}$ communication. In general, methods that receive state quantity information from the internal sensors of the 
preceding vehicle via $\mathrm{V} 2 \mathrm{~V}$ communication have higher accuracy than methods based on external sensors. Further, in previous studies, simulation-based evaluations have shown that CACC yields higher control performance with lower feedback gain than ACC.

In this study, a CACC for small EVs is developed and evaluated in order to confirm the validity of the previously reported simulation-based findings. Thus, a driving evaluation test using four small EVs with the developed CACC is conducted. The paper is organized as follows. In the next section, we briefly review related works on CACC and identify the technological issues. The CACC algorithm used in the small-EV evaluation is then presented in section 3. Section 4 describes the experimental procedure and results, and is followed by the conclusion.

\section{Related Works}

In this section, previous works related to the CACC examined in this study are discussed and the technological problems affecting this approach are identified. As explained in the Introduction, the primary technological problem affecting ACC technologies is the simultaneous achievement of both ride comfort and a stable V2V distance. During transfer of sensor information between vehicles, errors in the preceding-vehicle data may be amplified when transmitted to the following vehicle. The measure of the degree of V2V-distance error amplification is known as string stability; this is one of the more important indicators of longitudinal control performance. However, Hidaka et al. ${ }^{(1)}$ have reported that not all ACCs on the market achieve string stability. As poor string stability indicates V2V-distance error amplification, such control systems can cause traffic congestion or collisions.

In order to achieve string stability, the feedback gain of the V2V distance control must typically be enhanced. However, a higher feedback gain increases the control sensitivity to errors in the $\mathrm{V} 2 \mathrm{~V}$ distance. As the resultant behavior differs significantly from human driving behavior, this type of control tends to yield decreased ride comfort. The ACCs currently on the market prioritize driver acceptance; thus, improved ride comfort is provided at the expense of string stability. However, in order to realize a fully autonomous driving system, both string stability and ride comfort must be obtained simultaneously; this can be achieved using CACC.

In response to recent advancements in communication technology, CACC has been receiving increased research attention. For example, a CACC system has been presented and practically validated in a recent study by Naus et al. ${ }^{(2)}$. Note that two CACC-equipped vehicles were used for the validation in that work. Further, a simulation-based analysis on the robust stability and performance of closed-loop systems has been conducted, in which ACC and CACC technologies were compared ${ }^{(3)}$. The CACC used in that study was based on the sliding mode method. In addition, Omae et al. ${ }^{(4)}$ have reported a CACC design for trucks, along with an accompanying simulation-based evaluation, while $\mathrm{Xu}$ and $\mathrm{Raja}^{(5)}$ have analyzed the difference in behavior of ACC and CACC with regard to lane merging. Finally, Lei et al. ${ }^{(6)}$ have evaluated the influence of V2V communication packet loss on control.

Almost all of the technologies presented in the above studies were evaluated via simulation; thus, the validity of those evaluations must be reconfirmed using actual physical vehicles. Further, as regards the cited studies in which evaluations using actual vehicles were conducted, no more than three vehicles were used. However, in order to ensure that string stability is achieved satisfactorily, more than three vehicles should be employed. Therefore, the present study evaluates the technological validity of the CACC approach using four small EVs.

\section{Control Algorithm}

The CACC algorithm for small EVs developed in this study assumes a case in which a preceding vehicle is present. Because of this assumption, the longitudinal control strategy must be based on either a constant-distance gap (CDG) or constant-time gap (CTG) method. A CDG control strategy maintains a constant target $\mathrm{V} 2 \mathrm{~V}$ distance, whereas a CTG control strategy allows the target V2V distance to fluctuate in proportion with the vehicle's velocity. Thus, a CTG control strategy yields similar driving behavior to that of a human, and it is easier to ensure control stability. Therefore, the CACC developed in this study is based on CTG control strategy.

To incorporate CTG control strategy in the CACC, feedback on the preceding-vehicle acceleration, the relative velocity, and the $\mathrm{V} 2 \mathrm{~V}$ time are required. However, it is not appropriate to use the acceleration of the preceding vehicle directly in the feedforward expression of a CTG control strategy, or to use the velocity of the preceding vehicle directly in the relative velocity feedback expression. Therefore, a feedforward and feedback filter appropriate for a CTG control strategy was designed in this study. 


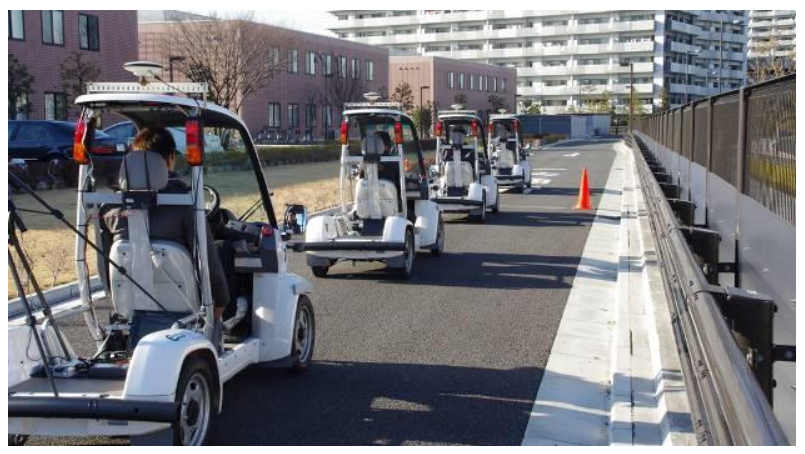

Fig. 1. Small EVs used in CACC experiment.

\section{Experimental Evaluation}

This section reports on an experimental evaluation of CACC string stability using the small-EV CACC algorithm presented in section 3 . The experimental conditions are first specified; then, the experimental results are reported.

\subsection{Experimental Conditions}

Four small EVs that could operate under the CACC described in section 3 were used for the experimental evaluation. The small EVs were organized in a line, as shown in Fig. 1, and labeled A-D, beginning with the first vehicle. The experiment was conducted on a flat, paved straight road, and each vehicle could share information with the nearest preceding vehicle only. A local area network (LAN) with 50-ms frequency was used for the inter-vehicle communication. In the experiment, the preceding vehicles transmitted their current accelerations, velocities, and synchronous data. Further, laser range finders (LRFs) were equipped on vehicles B-D, so as to measure the $\mathrm{V} 2 \mathrm{~V}$ distance between each nearest preceding vehicle with 50-ms frequency. The steering of each vehicle was operated manually by a human driver. Vehicle A was accelerated up to $20 \mathrm{~km} / \mathrm{h}$ before the start of the experiment. Three s after the beginning of the experiment, A was programmed to decelerate to a speed of $10 \mathrm{~km} / \mathrm{h}$. The responses of the other vehicles to this deceleration were monitored. Note that the maximum acceleration command of each vehicle was set to $1.5 \mathrm{~m} / \mathrm{s}^{2}$, so as to establish an upper limit. The experimental conditions are illustrated in Fig. 2.

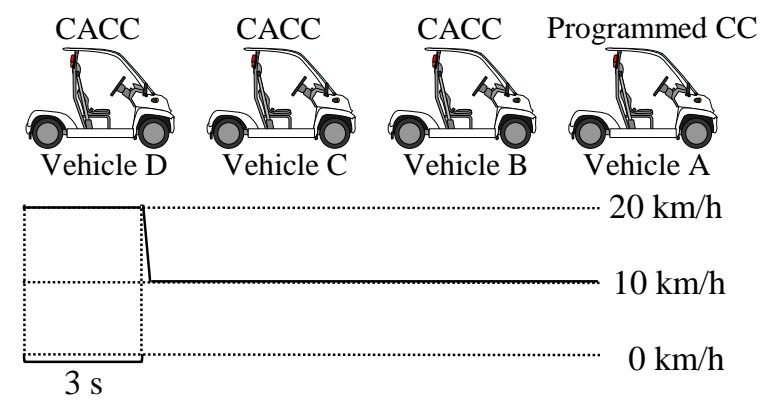

Fig. 2. Experimental Conditions.

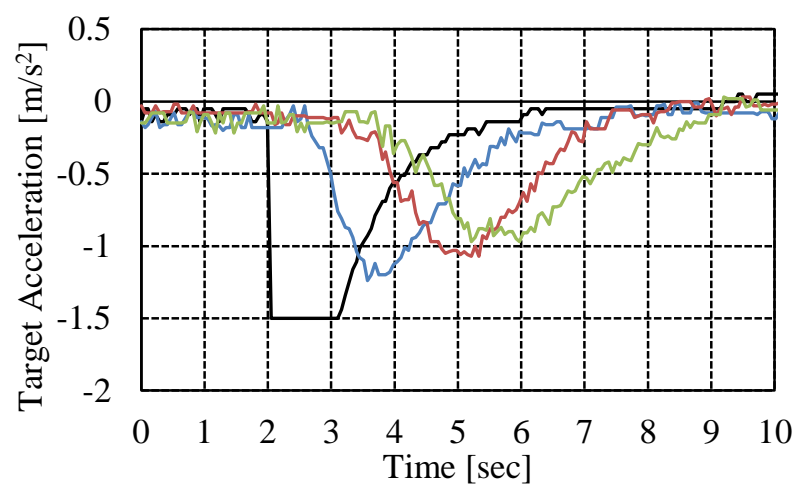

Fig. 3. Experimental results.

\subsection{Experimental Results}

Fig. 3 shows the experimental results. Hence, it is apparent that the acceleration command of $\mathrm{A}$ is attenuated when transferred to B-D. Thus, each control exhibits string stability. Moreover, improved ride comfort can be inferred, because no oscillation is exhibited in the acceleration commands of the following vehicles. These results confirm that CACC ensures both ride comfort and string stability. The responses of vehicles B-D are slower than their respective nearest preceding vehicles, because of the communication delay.

\section{Conclusions}

A CACC algorithm was developed and implemented on several small EVs and its performance was evaluated. In particular, the simultaneous realization of ride comfort and string stability was examined. A notable aspect of the physical evaluation was the use of more than three EVs (a total of four) to effectively evaluate the string stability; this is in contrast to previous practical evaluations. The CACC algorithm presented here employed designed feedforward (for the preceding-vehicle acceleration) and feedback (for the preceding-vehicle velocity) filters. The comparative 
experiment on the presence or absence of the designed filters using four small EVs, each with an LRF and communication device, confirmed that the control algorithm proposed in this study yields high following performance. Satisfactory ride comfort was also observed. In the future, we aim to conduct further studies targeting safety enhancement, with a view to accomplishing practical CACC.

\section{References}

(1) Ken Hidaka, Hironobu Kitaoka, Kenichi Kitahama, Mitsuhisa Shida, Hiroshi Fujimoto, Noriyuki Kisu, Hiroyuki Koike, Junji Eguchi, Hideki Kaseyama, and Tetsuya Kato : "Smooth Traffic Flow using ACC in Sag Section of Expressway", Transactions of Society of Automotive Engineers of Japan, Vol. 44, No. 2, pp. 765-770, 2013

(2) Gerrit J. L. Naus, René P. A. Vugts, Jeroen Ploeg, Marinus J. G. van de Molengraft, and Maarten Steinbuch : "String-Stable CACC Design and Experimental Validation: A Frequency-Domain Approach", IEEE Transactions on Vehicular Technology, Vol. 59, No. 9, pp. 4268-4279, 2010

(3) Xiao-Yun Lu, J. Karl Hedrick, and Michael Drew : "ACC/CACC-Control Design, Stability and Robust Performance", Proceedings of the 2002 American Control Conference, Vol. 6, pp. 4327-4332, 2002

(4) M. Omae, T. Ogitsu, R. Fukuda, and W. Chiang : "Longitudinal Control Algorithm for CACC (Cooperative Adaptive Cruise Control System) of Heavy-Duty Vehicles", Transactions of Society of Automotive Engineers of Japan, Vol. 44, No. 6, pp. 1509-1515, 2013

(5) Qing $\mathrm{Xu}$, and Sengupta Raja : "Simulation, Analysis, and Comparison of ACC and CACC in Highway Merging Control", IEEE Proceedings of the Intelligent Vehicles Symposium 2003, pp. 237-242, 2003

(6) Chenxi Lei, Emiel Martijn van Eenennaam, Wouter Klein Wolterink, Jeroen Ploeg, Georgios Karagiannis, and Geert Heijenk : "Evaluation of CACC String Stability using SUMO, Simulink, and OMNeT++", EURASIP Journal on Wireless Communications and Networking, pp. 1-12, 2012 